(Aus dem physiologischen Laboratorium in Utrecht.)

\title{
Ueber Reflexe von der Herzkammer auf das Herz des Frosches.
}

Von

\section{Dr. L. J. J. Muskens.}

\section{Einleitung.}

Im Gegensatz zu der äusserst reichen, noch jedes Jahr heranwachsenden Litteratur über die Bewegungen des Herzens, die Eigenschaften des Herzmuskels und dentEinfluss von centrifugalen Nerven auf die Herzbewegung ist uber $r_{a t}^{2}$ die Sensibilität des Herzens, über centripetale, rom Herzen ausgehende ,Nerveneinflüsse überhaupt, nur Weniges veröffentlicht worden. Im Besonderen ist die Frage, ob die Herzbewegung selbst vom Herzen aus auf reflectorischem Wege direct beeinflusst werden könne, noch höchst ungenügend behandelt worden. Dass bei Säugethieren Reflexe von der Kammer auf das Herz vorkommen, darf zwar nach den Beobachtungen und Versuchen von Wooldridge und $\mathrm{Z}$ wa a rde make r nicht mehr bezweifelt werden, aber wie und wo diese Reflexe zu Stande kommen, ob es sich um peripherische oder centrale Reflexionen handelt, ob sie auch bei den Herzen anderer Wirbelthiere bestehen, diese und viele weitere Fragen sind noch gänzlich ungelöst.

Es liegt nahe, gerade beim Herzen, allgemein, sehr hoch entwickelte nervöse Einrichtungen zu automatischer Modificirung und Regulirung der eigenen Thätigkeit auf reflectorischem Wege zu erwarten. Finden wir solehe Einrichtungen doch sehr verbreitet bei anderen musculösen Organen - es sei nur an den Reflextonus der gewöhnlichen Muskeln erinnert - und ist doch das Herz von allen anderen Organen des Körpers her auf die. mannigfachste Weise durch Nervenreflexe direct zn beeinflussen. Es wäre höchst merkwürdig, wenn vom Herzen selbst ans eine derartige Beein- 
flussung nicht, und zwar nicht in besonders wirksamer und vielseitiger Weise möglich wäre.

Die folgende Untersuchung soll diese und die daran sich zunächst anschliessenden Fragen für den Frosch, als das Hauptversuchsobject der Herzphysiologie, zu beantworten versuchen. Gelang: es bei diesem, das Vorhandensein von Einrichtungen der erwähnten Art nachzuweisen, so durfte deren Existenz bei den höheren Vertebraten und dem Menschen a fortiori angenommen werden und eröffnete sich damit die Aussicht auf einen tieferen Einblick in das verwickelte Getriebe des normalen Herzmechanismus, damit aber auch weiter in das Wesen und den Ursprung compensatorischer Processe, wie sie in pathologischen Zuständen im Herzen so vielfach sich abspielen und dank ihrer Zweckmässigkeit ein weiteres Fortbestehen des Individuums auch bei tiefgreifenden Störungen des Herzens ermöglichen.

Schon gelegentlich seiner neneren Untersuchungen über die Herzthätigkeit (dies Archiv Bd. 52, 56, 59, 61, 62, 65) hatte Prof. Engelmann beiläufig eine ziemlich grosse Anzahl von Versuchen zur Beantwortung jener Fragen angestellt. Aus diesen nicht publicirten Experimenten hatte sich ergeben, dass nach Zermalmung von Hirn und Riickenmark genau auf die Kammerspitze localisirte electrische, chemische, thermische, mechanische Reize bei Fröschen (R. temporaria und esculenta) niemals Reflexe auf das Herz veranlassen. Weil aber dieses Resultat negativer Art war und die Anzahl der angestellten Versuche mit Hinsicht hierauf und anf die vielen complicirenden Umstände nicht genügend gross ersehien und ausserdem anch bei erhaltenem Hirn und Ruickenmark bei vielen Individuen nur negative Ergebnisse erhalten wurden, erschien es Prof. Eng el man n wünschenswerth, durch eine eingehende Untersuchung an sehr reichem Material, unter sebr verschiedenen Umständen, die Frage womöglich endgiltig zu entscheiden. Seiner Aufforderung, diese Untersuchung auszuführen, bin ich nachgekommen und erlaube mir nun die Beschreibung und Ergebnisse der Experimente auf folgenden Zeilen mitzutheilen.

\section{Geschichtliches.}

Die bisher vorliegenden Thatsachen, aus denen das Bestehen von sensiblen Herznerven und die Möglichkeit von Reflexen von 
Herz auf Herz abgeleitet werden können, sind theils anatomischer, theils physiologisch-experimenteller, theils klinischer Art. Nach einander wollen wir diese Thatsachen besprechen und ibre Beweiskraft untersuchen.

a. A natomi sche Thatsachen. Makroskopisch-anatomische Forschung zeigte schon früh, dass Herznerven beim Menschen und anderen Vertebraten nicht pur im Muskelfleisch (Myocard), sondern namentlich auch im Peri- und Endocard sicb verbreiten. Man war geneigt, diese Nerven als sensibel zu betrachten, eine Auffassung, der in den letzten Jahren durch die mikroskopischen Untersuchungen über die Verbreitung und Endigungsweise der nicht fuil den Muskel direct bestimmten Nerven neue Stützen verliehen wurden. Um von anderen, weniger überzeugenden Untersnchungen zu schweigen, wiesen Tumaenzew und Dogiel (1) mit der Methylenblau-Methode feine subendotheliale Nervengeflechte in der Atrio-Ventricular-Klappe des Frosches nach, und Heymans und Demoor (2) bestätigten diesen Befund mit der. Methode von Golgi. Auch sahen sie in der Spiralklappe des Bulbus arteriosus einen ähnlichen feinen Plexus. Die aus den Nervengeflechten stammenden terminalen Fibrillen schienen ihnen im Protoplasma der Endothelialzellen der Klappen zu endigen. Sie halten sie für sensibel und meinen, dass sie reflectorisch einen regulatorischen Einfluss auf die Herzthätigkeit ausüben. Ohne diese Möglichkeit bestreiten zu wollen, darf man doch nicht den Gedanken an trophische (also centrifugale) Funktionen dieser endocardialen Nerven ohne Weiteres verwerfen; deuten doch viele Untersuchungen ${ }^{1}$ ), neuerdings wiederum die im Laboratorium von Prof. C. Winkler angestellten Versuche, entschieden auf einen trophischen Einfluss des N. vagus auf Myo- und Endocardium hin (J. P. Elias) (3), wie derselbe zavor schon für den $\mathrm{N}$. ischiadicus an der Arteria tibialis postica demonstrirt war (H. Bervoets) (4).

Diese Möglichkeit ist auch von H. S mirnow (5) erwogen worden, der unlängst die sensiblen Nervenendigungen im Herzen von Amphibien und Säugethieren einer gründlichen Untersuchung (Methylenblau-Methode) unterworfen hat. Bei Amphibien sah er

1) Vgl. die Literaturangaben bei $\mathrm{Th} . \mathrm{W}$ Engel mann, Ueber den myogenen Ursprung der Herzthätigkeit u. s. w. Dies Archiv Bd. 65. 1897. p. 571 . 
dicke, isolirt verlaufende markhaltige Fasern, zum Theil von den Bidder'schen Atrio-Ventricularganglien herkommend, unter wiederholter Abgabe von markhaltigen und markfreien Fasern schliesslich in Bäumchen feinster variköser Fasern endigen, und zwar im Bindegewebe des Ventrikel-Exocardiums, namentlich im oberen dritten Theil der Kammer. Bei Säugethieren fand er, ausser einem zum grössten Theil aus markhaltigen Nervenfasern gebildeten Netz und darans hervorgehenden endocardialen, sub- und intraendothelialen Nerven, eigenthümliche Endigungen, welche mit den ron Golg $i$ in Sehnen von gewöhnlichen Maskeln entdeckten, reich verzweigten Endbäumchen grosse Aehnlichkeit zeigen, und im Bindegewebe, namentlich des Endocardiums der Vorkammern, vor allen an der Umschlagstelle rom Septum, gelegen sind; weiter anch - jedoch viel weniger zahlreich - ahnliche Endigungen im Endocardium des oberen Theils des Ventrikels auf den Chordae tendineae und im Exocardium von Kammer und Vorkammer.

Nach Durchscheidung des einen $\mathrm{N}$. vagus bei einem Kaninchen, waren wenige, und 4 Tage nach Durchschneidung des rechten Vagus und linken Depressor bei einer Katze, alle Endbäumchẹn im Endocard degenerirt oder, unsichtbar geworden, während übrigens die Nerven vorzïglich gefärbt erschienen. Die hierauf von S mirnow gegriundete Vermuthung, dass die beschriebenen Endbäumchen Endigungen des N. depressor sein möchten, scheint uns nicht genügend motivirt um Gewicht daranf zu legen; gewiss aber wird man Smirnow zugeben müssen, dass die sensible oder wenigstens centripetale Funktion jener merkwürdigen Endigungen durch ihre Lage im Bindegewebe im allerböchsten Grade wahrscheinlich gemacht wird.

En twi c kel u ng ge s chichtlich e Gründe für die sensible Funktion der Herzganglien sind neuerdings von $\mathrm{H}$ is (6) und Romberg (7) beigebracht worden. Sie zeigten, dass die intracardialen Ganglien aus demselben embryonalen Zellenstrang als die Ganglia spinalia ibren Ursprung nehmen. Diese gemeinsame Anlage kanninzwischen, wie schon von verschiedenen Seiten bemerkt worden ist, kein zwingendes Argument für die Identität der Funktion von intracardialen und spinalen Ganglien abgeben; denn die gemeinschaftliche Anlage beider braucht ja nicht schon physiologisch determinirt zu sein.

b. Physiologische Thatsachen. Lange, sehr lange hat 
die alte Beobachtung, dass durch mechanische oder electrische Reizung Contractionen des Herzens ausgelöst werden können, ais Beweis für das Bestehen centripetaler Herznerven gegolten. Man betrachtete die Zusammenziehung als einen Reflexvorgang und suchte deshalb nach Reflexcentren im Herzen, die denn auch bald in den von $\mathrm{R}$ emak, L u dwig, Bidde r u. a. im Herzen entdeckten Ganglien gefunden zu sein schienen. Die Thatsache an sich - dass das Herz sich bei kuinstlicher Reizung seiner Wand zusammenzieht - beweist jedoch nichts. Sie kann, in Ueberein. stimmung mit der schon von Albrecht von Haller entwickelten Lehre von der Reizbarkeit der Muskelfasern, durch directe Erregung der netzförmig zusammenhängenden Muskelfasern erklärt werden. Diese Erklärung würde freilich ungenügend sein, wenn bei lokaler Reizung der Herzwand die Contraction nicht an der direct gereizten Stelle, sondern in gewisser Entfernung davon anhiibe; eine Behauptung, welche von mehreren Autoren aufgestellt worden ist. Schon Kü rschner (8) und Budge (9) wollen bekanntlich beobachtet haben, dass das Herz, wo auch gereizt, immer mit einer Vorkammer-Contraction $\left(A_{s}\right)$ anfängt, der dann eine Kammersystole $\left(V_{s}\right)$ folge. Eben so entschieden ( 8 und 9 ), wie diese Behauptungen ausgesprochen wurden, ebenso sicher sind sie falsch. Kein späterer Beobachter hat sie bestätigt. M. Schiff (10), später Engelmann, Gaskell u. a. sahen die Contraction immer von den gereizten Stellen ausgehen und von dort aus peristaltisch, resp. antiperistaltisch sich verbreiten. Damit in Uebereinstimmung ist die von Enge lmann (11) und Marehand (12) gefundene und von $\mathrm{Burdon}-\mathrm{S}$ anderson und anderen bestätigte Thatsache, dass die die Erregung begleitende electromotorische Wirkung immer an der gereizten Stelle anbebt und sich von hier aus nach allen Richtungen hin im Herzen verbreitet, und zwar mit derselben sehr geringen Schnelligkeit wie die Contractionswelle. Die antiperistaltischen, von $V$ nach $A$ fortschreitenden Contractionen, welche man durch Reizung der $V$-Spitze auslösen kann, sind beim frischen blutdurchströmten, wie beim ausgeschnittenen Herzen, mit der Methode der einfachen oder doppelten Suspension $(13,14,15)$ registrirt worden, und damit die Möglichkeit eines Irrthums, an den bei blosser Inspection noch allenfalls gedacht werden könnte, völlig ausgeschlossen.

Yon grösserer Wichtigkeit und sebr zu Gunsten centripetaler 
Nerven, ja zu Gunsten intracardialer Reflexe schien die Beobachtung Volkmann's (16) zu sprechen, der, nach unvollkommener Spaltung der Kammer von der Spitze nach der Basis zu, bei Reizung der einen Hälfte zwar auch die andere sich zusammenziehen sah, aber nicht umgekehrt. Dergleichen Fälle sind später auch von Engelmann (13) beschrieben worden. Er nahm jedoch jenen Thatsachen ihre Beweiskraft für das Bestehen intracardialer Reflexe $(17,18)$, indem er zeigte, dass anch bei gewöhnlichen und selbst bei curarisirten, deshalb dem Nerveneinfluss entzogenen Muskelfasern die normale reciproke einer irreciproken Leitung Platz machen kann. Der, wenn er richtig wäre, wichtige Fund Paglia n i's (19), der durch mechanische Reizung ausschliesslich des Eetocardiums beim Frosche reflectorische Contractionen ausgelöst haben wollte, während dies bei directer Reizung des Muskelgewebes nach Wegnahme des Ectocards nicht gelang, wurde gleichfalls von Engelmann entkräftet. Er wies darauf hin, wie schwer es sei, das äusserst dünne Ectocard der Herzspitze obne Miterregung der darunter gelegenen Muskelu zu reizen, und dass es unmöglich ist, diese Membran ohne Beleidigung des davon bedeckten Muskelgewebes abzupräpariren. Bei vorsichtiger Reizung des Ectocards der Spitze vollkommen frischer, sehr reizbarer Kammern mit einer sehr feinen Pincette beobachtete er ausserdem öfters keine Contraction, welche aber immer erfolgte, wenn die Pincette auch die Muskelfasern eingeklenmt hatte. Neuerdings hat Kaiser den Pagliani'schen Versuch bestätigen zu können gemeint und giebt an, dass man namentlich bei Herzen, die durch Ligatur der Aorta blutüberfüllt sind, ab und zu - nicht immer - bei Reizung der V-Spitze die Contraction an der V-Basis anfangen sehe. Wer den Versuch nachgemacht hat, wird inzwischen zugeben miissen, dass es nicht möglich ist durch blosse Inspection eine sichere Ueberzeugung zu gewinnen. Bei der wenigstens $100 \mathrm{~mm}$, vermuthlich mehr betragenden Fortpflanzungsgeschwindigkeit im frischen blutdurchströmten Kammermuskel und der Kleinheit des Froschherzens würde die Zeitdifferenz zwischen Anfang der Contraction an der V-Basis und an der V-Spitze nur höchstens 0.1 " betragen können; ein Intervall, zu klein, um unter den vorliegenden Umständen durch einfache Betrachtung mit dem Auge eine Entscheidung über den Ort des ersten Auftretens der Contraction zu gestattẹn. Auch können spontane, von der Basis ausgehende Con- 
tractionen bier sich störend cinmischen, za deren Entstehung die übermässige Ausdehnung der Herzenswand durch das gestaute Blut leicht Anlass geben könnte. Sobald die Leitungsfähigkeit soweit abgenommen hat, dass Spitze und Basis sich merklich ungleichzeitig zusammenziehen, ist immer nur zu sehen, dass die Contraction an der direct mechanisch gereizten Stelle, gleichviel wo sie liege, anfängt. Später bat $\mathrm{K}$ a is er $(21,22,23)$ verschiedene $\mathrm{Be}$ obachtungen über Interferenzen von künstlichen und natürlichen oder zwischen verschiedenen künstlichen Reizungen des Herzens mitgetheilt, durch welche er intracardiale Reflexe glaubt nachweisen zu können. Insoweit diese Angaben richtig sind, haben sie schon frïher in den Engelmann'schen Experimenten $(14,15,16)$ ibre Erklärung gefunden, ohne dass es notbwendig oder auch nur zulässigg wäre, dabei die Intervention centripetaler oder centrifugaler Nerven in der Herzwand anzunehmen. K ai ser's hauptsächlichstes Argument beruht darauf, dass auch der isolirte $\mathrm{V}$ eine compensatoriscbe Rube nach einer Extrasystole bei "continuirlicher" Reizung zeigt, und zwar in derselben Weise als der spontan und normal sich contrahirende $V$. In erster Linie hat er aber den Beweis nicht geliefert, dass in den von ihm mitgetheilten zwei Fällen die Reizung wirklich continuirlich war. Im Gegentheil, man hat Reeht anzunehmen, dass die Reizung periodisch war und, wie in der Norm, von der Vorkammer ausging; denn Ka i se r (23) sehnitt in dem einen Versuch (Vers. 15, II, 1895, Tab. IX) „dicht o be r h a l b der A-V-Grenze", in dem anderen (Vers. 9, I, 1895, Tab. X) „i n der A-V.Grenze" durch. Im ersteren Fall waren deshalb gewiss noch Vorkammer-, vielleicht auch Sinusreste in Zusammenhang mit $\mathrm{V}$ beigeblieben, im zweiten war ersteres wenigstens wahrscheinlich der Fall.

Ausserdem lehrt ein Blick auf die von $\mathrm{K}$ ai ser mitgetheilten Zahlen und Abbildungen (a. a. O. Taf. VI, Fig. 7 und 8), dass, seiner Bebauptung entgegen, die "compensatorische Ruhe" in seinen Versuchen keineswegs die Regelmässigkeit besass, wie beim normal pulsirenden Herzen. Während sie in der Norm (15) immer mit einer fast mathematischen Genauigkeit das vorhergehende Deficit compensirt, ist ihre Unregelmässigkeit in $\mathrm{K}$ a i ser's Versuchen derart, dass sie in seinen Fillen nicht den Namen "compensatoriseher" Ruhe beanspruchen kann. Anstatt dem doppelten Betrag der gewöhnlichen Periodendauer (T) gleich zu sein, 
Ueber Reflexe von der Herzkammer auf das Herz des Frosches. 335

ist die Summe der Dauer der Extraperiode and der vorhergehenden abgekuirzten Periode in K.'s Fig. 7 u. a. $1.7 \mathrm{~T}$, in Fig. $7 \mathrm{a}$ u. a. $1.8 \mathrm{~T}$, in Fig. 8 1.5, 2.4, 2.1 T. u.s.w.

Dazu ist K. in den Fehler verfallen, die Extrasystolen in zu kurzen Intervallen einander folgen zu lassen, in Intervallen nämlich, in welchen meistens nur 2, höchstens 4 gewöhnliche Systolen stattfinden konnten (Fig. 7, 7a und 8).

Bei einer so mehrfach incorrecten Einrichtung der Versuche hat man ihnen jeglichen Werth zur Entscheidung unserer Frage abzusprechen, um so mehr, als alle Thatsachen ohne Annahme intracardialer Reflexe ganz einfach aus den bekannten Eigenschaften des Herzmuskels erklärt werden können. Die wenigen Ausnahmen, welche, unter Tausenden von Fällen, Prof. Engelmann und auch ich fanden, sind von Ersterem zur Geniige aufgeklärt worden (u. a. 15, p. 334 u. flg. s. a. 36, p. 145 flg.). Andere Versuche oder Thatsacben, welche als Beweise für das Vorkommen intracardialer Reflexe gelten könnten, sind uns nicht bekannt.

Ist deshalb das Bestehen. sensibler oder centripetaler Nerven der Herzkammer, welche direct die intracardialen Centren beeinflussen könnten, nicht dargethan, unzweifelhaft ist es, dass Reize

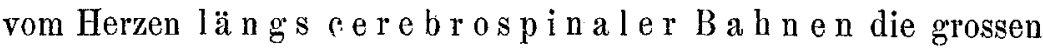
Nervencentren erreichen und hier Reflexe auf das Herz selbst auslösen können, ähnlich wie auf andre Muskeln. Der ex perimentelle Beweis auch für diese Art von Sensibilität des Herzens ist erst vor Kurzem geliefert worden.

Die Goltz'schen Versuche $(24,25)$, im Anschluss an ältere Experimente von Budge unternommen, scheinen für die Sensibilität des Sinus des Froschherzens beweisend, wiewohl die von ihm angewandte chemische Reizung der Herzwand mit Essigsäure die Möglichkeit, dass sensible Nerven von anderen benachbarten Theilen mit gereizt wurden, nicht ganz ausschliesst. Er weist darauf hin, dass Reizung mit einer glïhenden Nadel lange nicht so constante Resultate gab und auch, dass durch Reizung der Vorkammern nur schwer, höchst selten durch Reizung der Kammer Reflexe ausgelöst werden konnten. Am leichtesten erhielt Goltz Reflexbewegungen des Thieres durch Application einer sauren Flüssigkeit anf die Hinterfläche des Herzens, da, wo die Venae cavae zum Sinus zusammenfliessen. Das ist die 
Gegend, wo beide Vagi in das Herz eindringen. Nach Durehschneidung beider Vagi wurde der Reflex nicht mehr beobachtet.

Bei 'einer jungen Katze wurden aber auch nach Durchschneidung beider Vagi "unzweideutige Reflexbewegungen des ganzen Körpers" gleich nach der Quetschung der Vorkammer mit einer kleinen Pincette von Goltz wahrgenommen. - Goltz' Angaben wurden für den Frosch von Gurboki (26) bestätigt, der auch bei Kaninchen Reflexzuckungen (der Hinterbeine) sah, jedesmal wenn „die Hinterfläche der Vorhöfe mit einem in Essigsäure oder besser in Schwefelsäure getränkten Schwämmehen gereizt" wurde, noch besser "vermittelst einer feinen Pipette, in die man ein paar Tropfen concentrirte Schwefelsäure eingesogen" hatte. Die Reflexe blieben nach Durchschneidung beider Vagi aus.

Die Entdeckung Cy o n's und Ludw i g's (27), dass durch Reizung des centralen Stumpfes ibres N. depressor Reflexe auf das Herz ausgelöst werden können, ist verschiedenen Deutungen zugänglich; blieb doch bis jetzt der exacte anatomische Beweis aus, dass die sensiblen Depressorfasern wirklich von der Herzwand herstammen; bis in die Kammer sind diese Fasern bis jetzt nicht verfolgt. Die beiden von S mirnow angefithrten Ver. suche (siehe oben) können nicht als strenger Beweis gelten.

Dass die Kammern des Hundes und des Kaninchens sensible Nerren besitzen, wurde, unabhängig von einander, von $\mathrm{Z}$ w a a $\mathrm{r}$ demaker (28) und Wooldridge (29) gezeigt. Die Störungen, welche Ersterer (1. c. p. 74) in Folge von Bertihrung mit einem stumpfen Instrumente wahrnahm - ansehnliche Verlängerung der zwei folgenden Herzperioden -, sind schwerlich anders denn als Reflexe zu deuten. Wooldridge sah bei elektrischer Reizung der centralen Stümpfe verschiedener Zweige der vorderen Kammernerven von Hunden Pulsverlangsamung mit Erhöhung des arteriellen Druckes. Wenn hier Stromzweige auf centrifugale Herznerven wirklich gänzlich ausgeschlossen waren, ist der Beweis geliefert.

Unaufgeklärt aber bleibt, dass in so vielen Fällen nach dem einstimmigen Zeugniss aller Experimentatoren tiefe mechanische Verletzung oder Reizung der Herzkammern keine andern Störungen veranlasst, als solche, welche auf directer vorübergehender Reizung der Muskelsubstanz beruhen, eigentliche Reflexe oder Andeutungen von Sensibilität aber gänzlich ausbleiben. Speciell auch fiir den 
Ueber Reflexe von der Herzkammer auf das Herz des Frosches. 337

Menschen gilt dies. Wir erinnern nur an die Fälle des Grafen Montgomery, den $\mathrm{Har}$ vey (30), und namentlich der Frau Seraphin, den von Zjemssen (34) gründlich untersucht und beschrieben hat, wo bei keinerlei Reizung des naheza blossliegenden Herzens irgendwelche Empfindung: im Herzen erregt werden konnte.

Viel weniger Werth als diese physiologischen Versuche besitzen die

c) Klinischen Beobachtungen über Sensibilität des Herzens. Vielfach werden, wie bekannt, bei Herzkrankheiten ausser den mechanischen Störungen der Funktion auch Sensibilitätsstörungen in der Herzgegend wahrgenommen: Schmerz, welchen der Patient im Herzen selbst oder wenigstens in der Herzgegend localisirt, entwẹder isochron mit dem Herzschlag kommend und gebend, oder mebr continuirlich und in Anfällen auftretend. Ein sehr bekannter und charakteristischer Complex von derartigen krankbaften Sensationen ist die sogen. Angina pectoris. Im Allgemeinen wird die Erklärung jener Sensibilitätsstörung gesucht in patbologiseher Reizung sensibler Herznerven, und die vielfachen "nervösen" Störungen in der Herzthätigkeit (Aenderungen in der Pulsfrequenz, Arhythmie u.s. w.) in Reflexen, welche von jenen Nerven ausgehen (siehe u. A. $\mathrm{H} . \mathrm{Huchard}$ [32] und A. Petit [33]). Gewiss wird man zugeben müssen, dass diese herrschende Auffassung viel Wahrseheinlichkeit hat, aber bei der Unmöglichkeit, durch subjective Wahrnehmung die Stellen, woher die primäre Reizung stammt, genau festzustellen - entweder von der Unggebung des Herzens (Herzbeutel z. B.) oder von der Herzwand selbst, und in letzten Fall von Vorkammer oder Kammer, vom Endo-, Myo- oder Ectocardium - dürfen wir die genannten Thatsachen nicht als unzweideutige Beweise für das Bestehen sensibler Nerven in der Herzwand betrachteñ.

\section{Eigene Untersuchnng.}

a) Einrichtung der Versuche.

Meine Versuche beziehen sich nur auf die Sensibilität der Herzkammer von Rana temporaria und esculenta, und zwar auf ihre Sensibilität für electrische Ströme, speciell für schnell auf 
einander folgende Inductionsströme. Wir beschränkten uns auf electrische Reizungen, nachdem es sich in vielen Versucben berausgestellt hatte, dass weder durch meehanische, noch durch chemische (Schwefelsäure, Salpetersäure, Essigsäure, Nitras Argenti) oder thermische Reize ein Vortheil zu erreichen war.

Strenge Localisation der electrischen Erregung auf die Kammerspitze wurde erreicht mittels des Kunstgriffes der Schwächung der extrapolaren Stromdichte (En ge 1 m a n n, Onderzoekingen etc. [4] IV, 1896, p. 107-109), indem man das ganze Herz mit einer gutleitenden unschädlichen Masse (Blutgerinnsel, diunne Gelatine mit 0,6\% $\mathrm{NaCl}$ ) umhüllte, aus der nur die Kammerspitze hervorsah, an welche dann zwei Electroden mit ziemlich scharfen Spitzen (Stecknadeln, mit Froschdiundarm uberzogen) und sehr kleiner intrapolarer Strecke (etwa $1 \mathrm{~mm}$ ) angelegt wurden. Ein hoher Wall von feuchter entfetteter Watte verhütete das Wegsinken der das Herz umhüllenden Gallerte. Der Ischiadicus eines sehr empfindlicben Froschpräparates konnte unter diesen Umständen an beliebige Stellen der Kammerbasis angelegt werden, ohne dass auch bei den stärksten der von uns verwendeten Induetionsströme (2 Groves im primären Kreis, ganz anfgeschobene Rollen) Zuckung erfolgt wäre.

Bei der Unzulänglichkeit und Unsicherheit der einfachen Beobachtung mit dem Auge war es nöthig, die Bewegungen des Herzens zu registriren. Da es uns nicht darauf ankam, Druckoder Volumschwankungen, sondern iiberbaupt nur Aenderungen der Herzthätigkeit zu beobachten und zu messen, durften wir uns auf die Suspensionsmethode beschränken, die ihre vielfachen Vorzüge aufs Neue bewährte. In vielen Fällen genügte die einfache Suspension der Kammerspitze; $a b$ und zu wurden die Bewegungen der Kammer und Vorkammer für sich, jede mit einem eigenen Hebel, registrirt in derselben Weise und mit denselben Instrnmenten, wie früber durch Prof. Engelman n (Pantokymogràphion in Verbindung mit dem rhythmischen Polyrheotom, Stimmgabel als Chronometer, electrisches Signal als Reizschreiber, vgl. dies Arehiv Bd. 52, 1892, S. 357 u. 603 ; Bd. 60, 1895, S. 28). Um Eigenbewegungen der Thiere thunlichst auszuschliessen, wurden die Frösche vor den Versuchen sehwach curarisirt. Man hat hierbei streng im Auge zu behalten, dass die Giftwirkung so schwach wie möglich, höchstens nur eben zur Läbmung der willkürlichen 
Muskeln genügend sei. Schon bei wenig grösseren Gaben wird die Reflexreizbarkeit des Herzens geschwächt oder gänzlich aufgehoben. Zur Schätzung des Grades der Curarisirung mag dienen, dass ein Thier, welches sebon innerhalb einer Viertelstunde nach Einspritzung völlig gelähmt war, für unsere Versuche im Allgemeinen nicht taugte. Selbst bei ungenügend curarisirten Fröschen gelang es oft nicht, Reflexe von der Kammer auszulösen, wo, in welcher Weise und wie kräftig man auch reizen mochte. Die meisten Aussichten für ein positives Resultat gaben die Frösche, bei welchen der "Klopfversuch ${ }^{*}$ von Goltz einen deutlichen Effekt anf das Herz hatte. Nichtsdestoweniger gab es auch unter diesen Individuen noch viele, von deren Kammer aus überhaupt keine Reflexe auf das Herz oder auf andre Organe erhalten werden konnten. Oefters mussten zehn und mehr Frösche geopfert werden, bevor ein geeignetes Object gefunden wurde. Auch balf es nichts, wenn wir die leicht curarisirten Thiere vorher ein oder zwei Tage mit freiliegendem Herzbentel unter einer feuchten Glocke aufbewahrten. Wohl schien dadurch - wie durch langes Blossliegen der Bancheingeweide nach Tarchan off - die Reflexreizbarkeit für den Goltz'schen Versuch erböht zu werden.

Künstliche Erhöhang der Reflexreizbarkeit mittels Einspritzung von Strychnin oder Durchschneidung des Hirnstammes oberhalb der Medulla oblongata wurde versucht, aber niemals mit gïnstigem Resultat.

b) B eschreibung der Ergebnisse.

Die Wirkungen, welche man bei kurzer Tetanisation der Ventrikelspitze oder der Ventrikelmitte beobachtet, lassen sich in zwei natürliche Gruppen bringen:

1. Oertlicbe, welche als directe Wirkungen der Ströme auf die Kammerwand zu betrachten sind.

2. Reflectorische, indirekte, welche Folge von Reizung centripetaler Nerven in und anf dem Ventrikel sind.

1. Oertliche Reizwirkungen. Hierher hat man zu rechnen: a) Die Extrasystolen, welche bei genügender Intensität des Reizes stets auftreten, falls die Reizung nicht in das refraktäre Stadium von $V$, also wesentlich in die Zeit der Kammersystole $\left(V_{s}\right)$ fiel. Diese Besehränkung deutet zugleich an, wie man bei Reizung die Extrasystolen vermeiden kann: indem man unmit- 
telbar vor oder während $\operatorname{der} V_{s}$ reizt. Dieser direkte Reizungserfolg kann in den Curven nieht missverstanden, d, i. als Reflex aufgefasst werden. Denn er tritt nahezu unmittelbar, höchstens 0,25 " nach der Reizung ein, während reflektorische Folgen immer eine längere Latenzzeit haben. Auf den Verlauf jener indirekten Folgen wird uibrigens durch die Extrasystolen im Allgemeinen kein Linfluss ausgeübt.

b) Aenderungen der Grösse und Kraft von $V_{s}$ (inotroper Einfluss). Die Aenderungen fanden fast immer nur in negativem Sinne statt. Die Schwächung war bei Reizung mit einem einzelnen Inductionsschlag oder sehr kurzem Tetanisiren schon bei der ersten der Reizung folgenden spontanen Contraction des $V$, d. i. innerhalb etwa einer halben bis 2 Sekunden, merkbar, erreichte ein Maximum nach 4 bis 10 Sekunden, nahm allmählich wieder ab, so dass die letzte geschwächte $V_{s}$ nach etwa 14 bis 40 Sekunden beobachtet wurde. Die Tabelle Ia giebt hiervon Beispiele: „Primum" giebt die Zeit an, welche verlief zwischen dem Beginn der Reizung und der ersten geschwächten $V_{s} ;$ "Maximum" die Zeit zwischen demselben Moment und der am meisten geschwächten $V_{s}$; "Ultimum“ die Zeit bis zur letzten merklich geschwächten $V_{s}$.

Tabelle Ia.

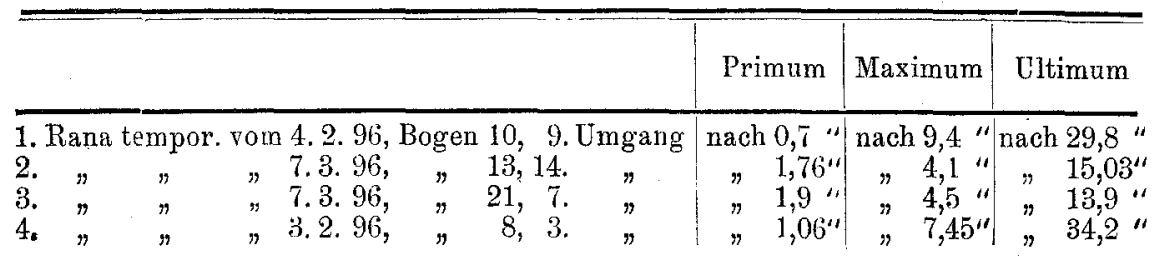

Tab. Ib giebt die Hubböhen der aufeinanderfolgenden $V s$ in den vier obengenannten Experimenten in Millimetern an, vor und nach Reizung der Kammer. Die erste Systole, welche dem Anfang der Tetanisation folgt, ist mit * angedeutet; die Daner der Tetanisation ist in Sekunden zwischen () angegeben.

Tabelle Ib.

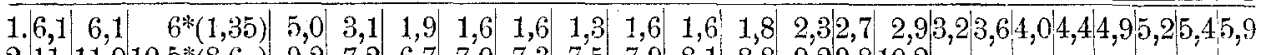

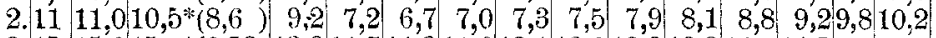

$3.1515,015 *(0,52) 13,911,711,211,612,113,013,213,914 \quad 14,5$

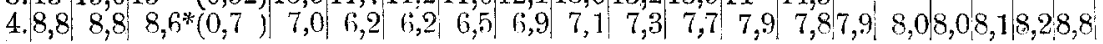


Man sieht hieraus, wie schnell der Effekt eintritt: schon innerhalb 0,7 Sekunden; und weiter, dass der Einfluss langsam ansteigt, langsam auch wieder vergeht. Wiewohl der Verlauf also mit den meisten Reflexen (s. später) darin übereinstimmt, dass das Maximum niebt direkt erreicht wird, kann dieser Einfluss doch nicht als Reflexwirkung betrachtet werden, weil e r a u ch an der isolirten ganglienfreien Kammerspitze wabrgenommen wird. - Die Phase der Herzcontraction, in welche die Reizung einfiel, schien auf den Verlauf der nichtreflektorischen $V_{s}$-Schwächung keinerlei Einfluss auszuüben. Sie trat speciell auch dann ein, wenn der Reiz nur während des refraktären Stadiums einwirkte. - Mit der Intensität und Dauer der Reizung wuchsen Grösse und Dauer der Wirkung innerhalb weiter Grenzen.

Oefters ist mir aufgefallen, dass der negativ inotrope Einfluss directer Reizung anf $V_{s}$, der beim blutdurchströmten Herz nicht deutlich war, sehr deutlich hervortrat im Verlauf längerer Suspension, und vor allem bei durch Blutverlust geschwächten Herzen. Im letzten Fall konnte die Schwächung bis zum Unsichtbarwerden der $V_{s}$ gehen. Ein anscheinend positiv inotroper Einfluss auf die $V_{s}$ wurde nur einige Male wahrgenommen, war aber sehr unbedeutend und beschränkte sich auch nur auf diejenige $V_{s}$, während weleher der Reiz einfiel.

2. R eflexerscheinungen. Diese sind theils Reflexe auf das Herz selbst, theils Reflexe auf andere Organe. Wir beschäftigen uns hier nur mit den ersten, den Reflexen von Herz auf Herz. Diese Reflexe sind sehr mannigfacher Art. Sie erstrecken sich auf die Vorkammern, auf die automatischen Apparate an den venösen Ostien und gelegentlich auch auf die Herzkammer selbst. Insoweit sie der Suspensionsmethode zugänglich sind, bestehen sie in Aenderungen:

1. der K r a f t, resp. der Grösse der Systolen : in o tr ope Effecte.

2. des T e mpos der Herzschläge: chronotrope Effecte.

3. der Leitungsfähigkeit der Muskelfasern : d r omotrope Effecte. 


\section{Nähere Beschreibung der Reflexe.}

Die drei beschriebenen Arten von Reflex auf das Herz kommen in manchen Fällen combinirt vor, gleichzeitig oder nacheinander; in anderen wird nur eine einzelne Art von Reflex, allenfalls combinirt mit einer zweiten, wahrgenommen. Diese Mannigfaltigkeit im Auftreten der Reflexwirkungen gilt auch für jedes einzelne Thier, wenn sein Herz unter verschiedenen Umständen gereizt wird. Vor allem ist die Intensität des Reizes hier von Einfluss, und zwar derart, dass im Allgemeinen bei schwächeren Reizen nur oder doch wesentlich nur negativ inotroper Einfluss auf $A$, bei kräftigeren Reizen auch chrono- und dromotrope Einfliisse merkbar sind. Auch isolirter chronotroper Einfluss wird wahrgenommen, jedoch selten; ausschliesslich dromotrope Wirkungen babe ich nie gesehen. Von 20 sorgfältig ausgemessenen Fällen kann ieh die folgende Uebersicht vorlegen.

\section{Tabelle II.}

Reflectorischer Effect von Reizung der Kammer auf:

\begin{tabular}{|c|c|c|c|c|}
\hline & Vorkammer & Kammer & Sinus & Leitung $\mathrm{A} \cdot \mathrm{V}$. \\
\hline $\begin{array}{r}I I \\
I I \\
I I I \\
I V \\
V \\
\text { VI } \\
\text { VII } \\
\text { VIII } \\
\text { IX } \\
\mathrm{X} \\
\mathrm{XI} \\
\mathrm{XII} \\
\mathrm{XIII} \\
\mathrm{XIV} \\
\mathrm{XV} \\
\mathrm{XVI} \\
\mathrm{XVII} \\
\mathrm{XVIII} \\
\mathrm{XIX} \\
\mathrm{XX}\end{array}$ & $\begin{array}{l}\text { inotr. - u. + } \\
\text { inotr. - } \\
\text { inotr. - } \\
\text { inotr. - } \\
\text { inotr. - u. + } \\
\text { inotr. - } \\
\text { inotr. - u. + } \\
\text { inotr. - } \\
\text { inotr. - u. + } \\
\text { inotr. - } \\
\text { inotr. - } \\
\text { inotr. - } \\
\text { inotr. - } \\
\text { inotr. - } \\
\text { inotr. - } \\
\text { inotr. - } \\
\text { inotr. - } \\
\text { inotr. - } \\
\text { inotr. - }\end{array}$ & $\begin{array}{l}\text { inotr. }+ \\
\text { inotr. }-u .+\end{array}$ & $\begin{array}{l}\text { chronotr. - u. + } \\
\text { chronotr. - } \\
\text { chronotr. - } \\
\text { chronotr. - } \\
\text { chronotr. - } \\
\text { chronotr. - } \\
\text { chronotr. - } \\
\text { chronotr. - } \\
\text { chronotr. - } \\
\text { chronotr. - } \\
\text { chronotr. - } \\
\text { chronotr. + u. - }\end{array}$ & $\begin{array}{l}\text { dromotr. - u. + } \\
\text { dromotr. - } \\
\text { dromotr. - ? } \\
\text { dromotr. - } \\
\text { dromotr. - } \\
\text { dromotr. - } \\
\text { dromotr. - }\end{array}$ \\
\hline
\end{tabular}

(Die Zeichen + und - geben an, ob der Einfluss in positivem oder in negativem Sinn stattfand.)

Die constanteste Reflexerscheinung ist $z$ weifellos die 
a. Inotrope Wirkung anf die Vorkammer. In 19 von den 20 ausgemessenen Fällen kam dieser Reflex zur Beobachtung, in 7 Fällen als einziger Erfolg. Der Effect war meist ausschliesslich negativ, d. i. die Grösse der $A$-Contractionen wurde reflectorisch geschwächt; selten wurde vor oder nach der Schwächung eine Verstärkung constatirt.

Der Verlauf der Reflexe war in den Fällen, wo andere Reflexwirkungen gleichzeitig fehlten, bei sehr kurzer, schwacher oder mässig starker Reizung folgender: Gleichviel, ob die Reizung in der Pause oder während $V_{s}$ oder $V_{d}$ einfiel und ob sie eine Extrasystole hervorrief oder nicht, immer wurde wahrgenommen, dass die erstfolgende, zu normaler Zeit einsetzende $A_{s}$ nicht oder nur wenig an Grösse eingebüsst hatte; die zweitfolgende $A_{s}$ war am meisten geschwächt, und die dritte oder vierte wieder normal. Auf nur eine oder zwei Perioden beschränkt sich die Wirkung nie. Doch mag dies bei noch schwächeren Reizungen vielleicht vorkommen.

In Tabelle III gebe ich einige Beispiele, in welchen unter „Primum“ die Zeit zwisehen Anfang der Reizung und Anfang der ersten geschwächten $A_{s}$ in Secunden angegeben ist; nnter ,Maximum" die Zeit zwischen Anfang der Reizung und Anfang der am meisten geschwächten $A_{s}$, während die letzte Spalte "Ultimum" die Zeit vom Beginn der Reizung bis zur letzten geschwächten As enthält.

T a belle III.

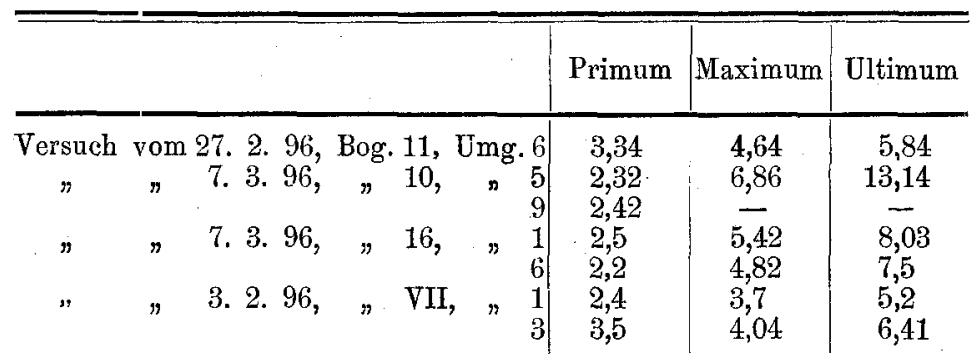

Wenn man sich nun erinnert, dass nach den Versuchen Nuel's (dies Archiv Bd. 9, 1874, S. 83) das Stadium der Latenz für die von

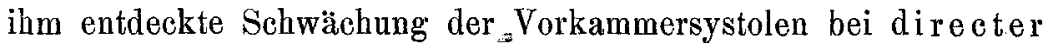
Reizung des Vagus des Frosches unter gewöhnlichen Umständen noch nicht $1 / 2$ Secunde dawert, so kann die reflectorische Natur des negativ inotropen Effectes in unseren Versuchen nicht bezweifelt 
werden. - Verglichen mit gewöhnlichen Reflexen auf willkürliche Muskeln ist die von mir gefundene Latenz von im Mittel 2"-3" enorm lang. Vermuthlich muss der Process der Reizung mehrere Neuronen passiren, oder aber es besitzen diese Neuronen eine specifisch grössere Trägheit.

Die Dauer des inotropen Einflusses auf $A$ ist ausserordentlich abbängig von der specifischen Reizbarkeit des Thieres und von der Intensität des Reizes. Was den Verlauf der Wirkung betrifft, so stellt sich aus der Tabelle heraus, dass das Maximum des Effectes keineswegs immer gerade in die Mitte zwischen Aufang und Ende des Effectes fällt; öfters aber sieht man Primum, Maximum, Ultimum in die resp. 2., 3. und 4. oder aber die 2., 4. und 6. Periode nach der Reizung fallen.

$a^{1}$. Inotroper. Effect a uf die Kammer. Dieser Einfluss kann positiv oder negativ sein, kommt aber selten vor. In der Tabelle III findet man ihn unter 20 Fällen 4 mal; in 3 Fällen positiv und negativ, in einem Falle bloss positiv. Der Effect ist nie von längerer Dauer; nach 2 oder 3 verstärkten $V_{s}$ können plötzlich eine oder mehrere geschwächte $V_{s}$ erfolgen.

Tabelle IV giebt einige Beispiele von Grösse und Verlauf der inotropen Effecte auf $V$. Die Zahlen geben die durch den Hebel 8-10 mal vergrösserten Hubböhen der $V_{s}$ in den aufeinander folgenden Perioden, vor und nach Reizung der Kammer in Millimetern an. Die unter $V_{s_{3}}$ stehenden Zablen deuten die erste Systole an, welche dem Anfang der Tetanisation folgt; die Dauer des Tetanisirens ist zwischen () dahinter angegeben.

Tabelle IV.

\begin{tabular}{l}
\hline \\
\hline
\end{tabular}

Dass diese Aenderungen in der Kraft von $V$ wirklich Reflexe sind, kann nicbt, bestritten werden. Die oben beschriebene, nicht reflectorische Schwäcbung der Ventrikelsystolen hat ja einen ganz anderen Verlauf und wird nach wie vor Vernichtung des Hirns und Rückenmarks wahrgenommen; zuweilen"trit sie sehon innerhalb 1 Secunde auf und verschwindet langsam; sie kann bis zu 
18 Secunden dauern. Die hier beschriebene $V_{s}$-Aenderung wird dagegen nie bei vernichteter Medulla gefunden, geht aber, wo sie vorkommt, immer mit anderen Reflexwirkungen zusammen; sie hat endlich eine Latenzdauer, welche mit jener der $A_{s}$-Schwächnng iibereinstimmt. Aus Aenderungen in der Dauer der vorhergegangenen Pausen kann der positiv inotrope Effect nicht erklärt werden.

b. Chronotrope Effecte. Diese wurden bei 13 von den 20 erwähnten Versuchsthieren angetroffen: in 12 Fällen combinirt mit inotropem (auf $A$ ) und dromotropem Effect auf $A V$. Ein einziges Mal nur fand sich der chronotrope Einfluss isolirt; die Latenzzeit vom Anfang der Reizung bis zur $A_{s}$ der ersten verlängerten Herzperiode zählte in diesem Falle in 3 Versuchen 2,77“, $3,8^{\prime \prime}$ und 3,9". - Im Gegensatz zum inotropen Reflex auf $A$ nahm ich hier kein Ansteigen bis zu einem Maximum wahr, sondern schon. die erste Periode war maximal verlängert; und nachher nahm auch der Effect nicht allmählich, sondern auf unregelmässige Weise ab, so dass nicht selten noch 2 oder $3 \mathrm{Mal}$ eine Verlängerung wie beim ersten Maximum erreicht warde.

In der folgenden Tabelle $\mathrm{V}$ findet man die Dauer $(T)$ der aufeinander folgenden Perioden, jede gerechnet vom Anfang einer $A_{s}$ bis zum Anfang der folgenden $A_{s}$; wäbrend unter $T_{Q}$ die Länge der Periode angegeben ist, in welche der Reiz einfiel; die Dauer der Reizung in Secunden ist in Klammern hinzugefügt. Die letzte Spalte „Latenz" giebt die Zeit an, welche zwischen dem Anfang der Reizung und dem Anfang der ersten verlängerten Penode verlief.

Diese Tabelle mag eine Vorstellung von der unregelmässigen Art und Weise geben, in welcher der chronotrope Effect bei der Vorkammer auftritt. Auch hier nimmt ibrigens, wie zu erwarten, der Effect mit der Dauer und der Intensität der Reizung an Dauer und Grösse zu.

In den Fällen Nr. 11 und 12 vom 18. 1.96 sieht man neben dem gewöhnlichen negativ inotropen Effect auch einen positiv chronotropen auf $A$ und zugleich, wie scheinbar launisch diese Effecte mit einander abwechseln können. Neben der sehr verkiurzten Periode von $0,88^{\prime \prime}$ sieht man eine sehr verlängerte von $3,26^{\prime \prime}$. 


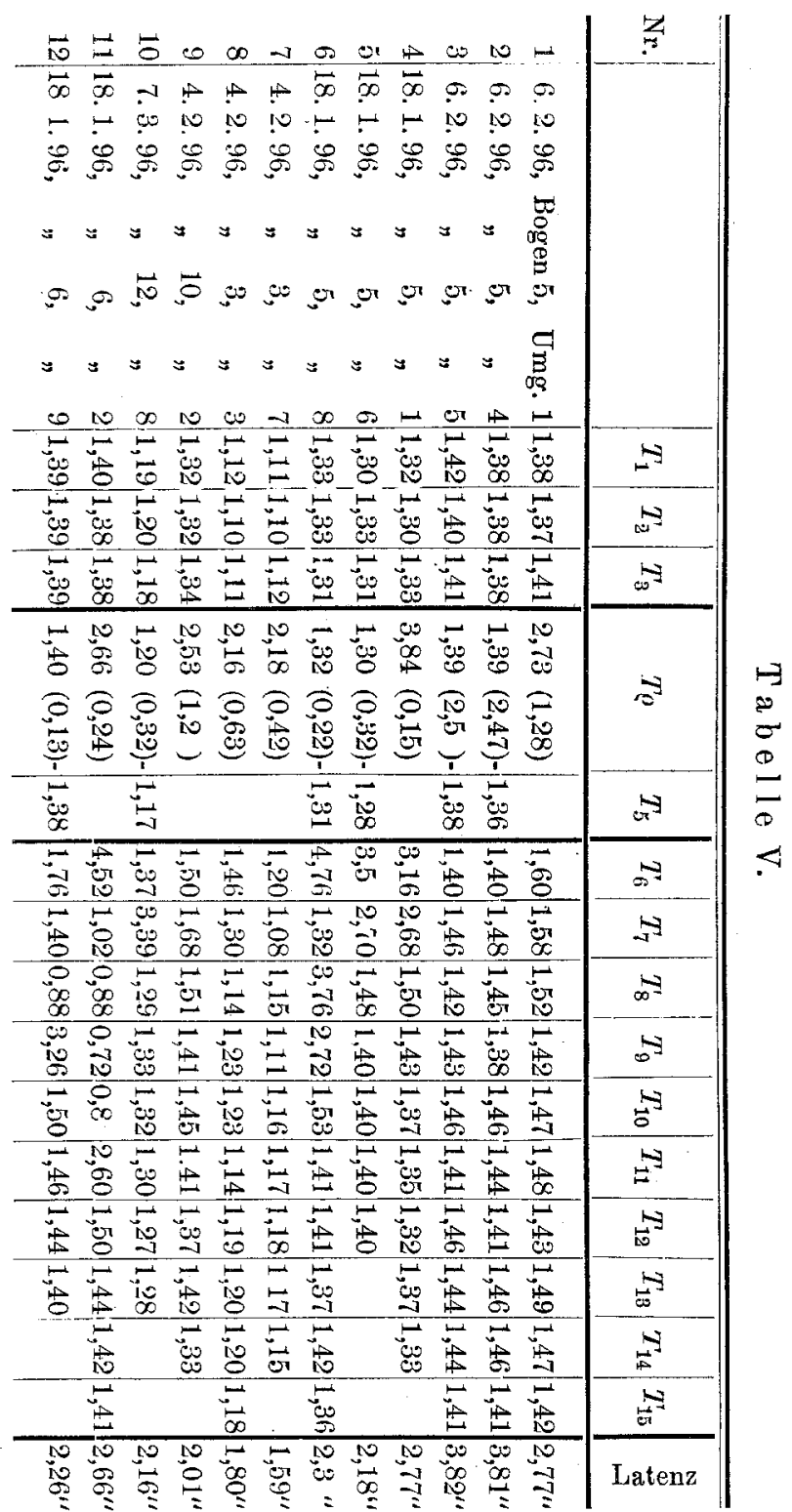

Es erübrigt mir noch die Erklärung der verhältnissmässig enorm verlängerten Perioden zu geben, welche man hier und da in der Tabelle wabrnimmt.

Wo die starke Verlängerung $T_{\varphi}$ betrifft (No. 1, 4, 7, 8, 9, 11), hat man es mit compensatorischen Pausen nach Extrasystolen 
zu thun; es ist zuweilen nicht möglich, diese beiden Perioden in den Curven für sich zu messen; deshalb wurden dann $T_{0}$ und $T_{5}$ zusammengenommen. - Bei den späteren Verlängerungen $T_{6}$ No. $4-6,11, T_{7}$ No. $4,5,10, T_{8}$ No. $6, T_{9}$ No. 6 und $12, T_{10}$ No. 11 handelt es sich nicht oder nur zum Theil um eine primär chronotrope Wirkung auf die automatischen Apparate an den venösen Ostien, sondern bauptsächlich um Ausfallen einer $A_{s}$ infolge Unterbrechung der Leitung von den venösen Ostien nach der Vorkammer, also um einen secundär chronotropen Effect.

Auch für den chronotropen Effect war ein Einfluss der Extrasystolen auf den Verlauf nicht nachweisbar.

c. Dromotroper Effect. Die Leitung von Sinus nach der Vorkammer, wie die Leitung von der Vorkammer nach der Kammer kann reflectorischen Aenderungen unterworfen sein. Nach neueren Versuchen von Prof. E $\mathrm{ng}$ e $\mathrm{lm}$ a $\mathrm{n} n$, in denen auch die Bewegungen der Venen und des Sinus messbar registrirt wurden, gilt dies auch für die Leitung von den Venen zum Sinus. Hierüber geben aber meine Curven, in denen die Sinusbewegungen nur ausnahmsweise, die Venenbewegungen nirgends sichtbar sind, keinen Aufschluss. - Die Aenderung in der Leitung fand stets in negativen Sinus statt; die von Gaskell bei Vagusreizung beschriebene Beschlennigung der Leitung wurde von mir nicht wahrgenommen.

Der negativ chronotrope Effect zeigt sich in der Curve in der Vergrösserung des Intervalles $A_{s}-V_{s}$, bezüglich des Intervalles $S i_{s}-A_{s}$. Die Leitungsabnahme geht zuweilen bis zur völligen Unterbrechung der Leitung. Ab und zu bekommt man den Eindruck, dass die Leitung von Sinus nach $V$ noch stattfand, während doch in der Curve nichts von $A$-Contraction zu bemerken ist. Diese Fälle müssen wohl im Anschluss an $\mathrm{Nuel}$, Gaskell und Engelmann in dem Sinne interpretirt werden, dass die Leitung durch $A$ zwar zu Stande kommt, dass aber wegen des gleichzeitigen, negativ inotropen Effects auf die Vorkammer, $A_{s}$ in der Curve nicht bemerkbar ist (14, S. $196 \mathrm{flg}$.). Bei guter Erregbarkeit und kräftigen Reizen kann während mehrerer Perioden die Contraction auf Venen und Sinus, beziiglich auf Venen, Sinus und Vorkammer beschränkt bleiben; die $A$-Contraction war im letzteren Falle aber immer auch geschwächt.

Zur richtigen Beurtheilung einer Periodenverlängerung von $A$ wird es somit nöthig sein, gleichzeitig die Bewegungen der 
grossen Venen und des Sinus messbar mit zu registriren, was in meinen Versuchen nicht geschah. Erst dann wird in jedem Falle mit Sicherheit zu sagen sein, in wie weit man es mit primär chronotropem. Einfluss zu thun hat, oder mit secundär chronotropem in Folge dromotroper Reflexe. Kann doch auch bei Beschleunigung der automatischen Thätigkeit an den venösen Ostien die Dauer der Vorkammer- und Kammerperioden verlängert sein (vgl. Engelmann, dies Archiv Bd. 65, S. 159-161 und Taf. VII, Fig. 25 und 26).

Noch ein anderer Umstand erschwerte die exacte Berechnung des dromotropen Ninflusses in hohem Grade. Bei der Anwendung: der einfachen Suspension ( $A_{s}$ und $V_{s}$ von demselben Hebel registrirt) liefert die Curve keine festen Punkte für genaue Bestimmung: des Anfangs der $A_{s}$ und $V_{s} ; A_{s}$ fängt öfters an in der absteigenden Linie der $V$-Diastole $\left(V_{d}\right)$, während $V_{s}$ oft ein wenig vor der Spitze $\operatorname{der} A_{s}$ einsetzt. Mehr Gewissheit bekommt man, wenn der Herzrevolution eine deutliche Pause vorangeht, und vor allem, wenn $A$ und $V$ jeder für sich registrirt worden sind. Auch dann nun stellt sich das Intervall $A_{s}-V_{s}$ in den oben beschriebenen Fällen selbst nach einer längeren Pause als ansehnlich verlängert heraus. Da nun nach den Bestimmungen Engelmann's $(14,35)$ das Intervall $A_{s}-V_{s}$ mit wachsender Dauer der Pause immer kürzer wird, ist also eine solche nach einer längeren Pause beobachtete Verlängerung des Intervalls $A_{s}-V_{s}$ nicht auf Ermidung durch die letzte vorausgegangene Contractionswelle zurückzuführen, sondern muss sie durch reflectorische, nervöse Einwirkung auf das Leitungsvermögen der $A$ und $V$ verbindenden Muskelbrücken erklärt werden.

Tab. VI giebt einige Beispiele des Verlaufs der dromotropen Wirkung. Die Zalulen bedenten (in Secunden) die Dauer des Intervalles zwischen $A_{s}$ und $\nabla_{s}$ jedesmal für idie drei der Reizung zunächst vorausgehenden und mehrere ihr folgenden Perioden. In der letzten Spalte ist wiederum die Zeit vom Anfang der Reizung bis zum Sichtbarwerden des negativ dromotropen Effects verzeichnet. - Die sehr lange Latenzdauer in No. 1 und 2 ist nur scheinbar. In diesen Fällen bewirkte die Reizung eine Extrasystole des Ventrikels und fiel deshalb die nächste spontane $V_{s}$ aus. Das Intervall $A_{s}-V_{s}$ konnte somit erst von der dritten $A$-Systole an gemessen werden. - Man bemerke auch in No. 2, 3 und 4 das 


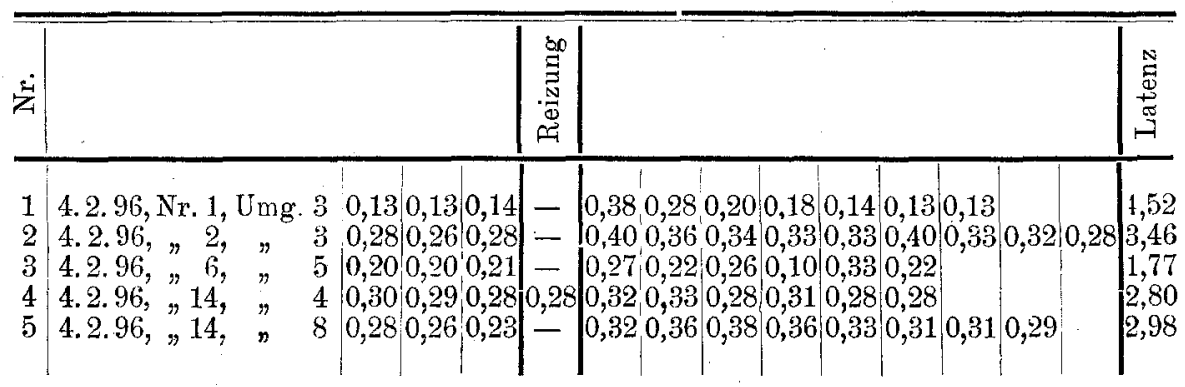

zweimalige Auftreten des Effects nach einmaliger Reizung. Die Wirkung ist sofort maximal und sinkt allmählich.

Es verdient noch Erwähnung, dass in meinen Versuchen negativ dromotrope nicht ohne gleichzeitige chronotrope Effecte vorkamen und dass auch der Verlauf beider Wirkungen in Allgemeinen parallel war.

\section{Die Centren der Herzreflexe.}

Wenn im Vorstehenden bewiesen wurde, dass von der Herzspitze aus Automatie, Contractilităt und Leitungsvermögen des Herzens auf reflectorischem Wege in sehr mannigfaltiger Weise beeinflusst werden können, so erhebt sich jetzt die Frage: Wo finden diese Reflexe statt; in den intracardialen Ganglien, iiber deren Function bis jetzt noch so wenig Sicheres bekannt ist? Oder in den grossen Centralorganen des Nervensystems?

Das Experimentum crucis erscheint einfach genug. Man zerstöre bei einem Frosche, bei welchem mit Sicherheit Reflexe vom Ventrikel aus zu erhalten waren, das Gehirn und Riickenmark und wiederhole die Reizung der Herzspitze. Findet man dann als constantes Resultat, aass der vorher constatirte Reflex erloschen ist, so bleibt kein Zweifel: im Riicken- oder verlängerten Mark kommt der Reflexbogen zu Stande.

Ich habe diesen Versuch zwanzig Mal angestellt. Das centrale Nervensystem wurde mittelst einer vom Schädeldach eingeführten kräftigen Nadel völlig zerstört, mit übrigens grösster Schonung des Herzens. Ausnahmslos waren unmittelbar nach der Operation die vorher sehr deutlichen Reflexwirkungen sämmtlich spurlos verschwunden und kehrten auch bei beliebig langem War- 
ten, trotz fortdauernder Circulation and übrigens ungesehwächter d i r e cte r Reizbarkeit der Kammer, nicht zurïck. Auch viel stärkere und länger währende als die zuvor gebrauchten wirlsamsten Reize waren erfolglos, selbst bei Application auf die Ventrikelmitte. Das Ergebniss ist also ganz dasselbe, wie nach den äusserst zahlreichen Messungen von Prof. Engelmann bei lokaler Reizung der Herzspitze am ganz ausgeschnittenen Herzen.

Zur näheren Veranschaulichung erlaube ich mir auf die in meiner Dissertation (34) gegebenen Abbildungen (Fig. 4 und 5), sowie auf die daselbst mitgetheilten ausführlichen Versuchsprotokolle zu verweisen.

\section{Schlussfolgerungen.}

Das Hauptresultat meiner Untersuchungen ist nach alledem: Beim Frosch existiren - und ohne Zweifel darf man dasselbe für die höheren Vertebraten vermuthen - Reflexe vom Ventrikel a uf das Herz, und zwar Reflexe sehr versehiedener Art und auf alle verschiedenen Herzabtheilungen. Diese Reflexe k ommen zu Stande, nieht in den Ganglienknotendes Herzens selbst, sondern ausschliesslich in den grossen Nervencentren, wahrscheinlich in der Medulla oblongata.

Kein einziges Factum ist bekannt, welches das Bestehen intracardialer Reflexcentra bewiese oder auch nur wahrscheinlich machte. Sollten sich bei weiteren Untersuchungen doch noch Erscheinungen finden lassen, welche zu Gunsten peripherischer Herzreflexe sprächen, so wäre immer noch an die von $\mathrm{Langley}$ hervorgehobene Möglichkeit einer Uebertragung der Erregung durch centripetale Leitung in peripherisch gereizten Zweigen centrifugaler Nervenprimitivfasern zu denken. Einstweilen erscheint es jedoch unnöthig, anf diese Möglichkeit näher einzugehen.

Wenn wir uns nach der Bedeutung der gefundenen Reflexe für das Individuum fragen, so lenchtet sofort ein, dass dieselben ein mächtiges Hilfsmittel für die Regulirung der Herzthätigkeit bei Störungen, welche die Kammerwand treffen, werden sein können.

Da Reflexe im Allgemeinen zweckmässig sind, d. b. die krbaltung des Individuums begïnstigen, so muss man dasselbe für 
die Reflexe von der Kammer auf das Herz erwarten; und zwar gewiss in besonders hohem Grade, da die Aufgabe, welche das Herz bei der Erhaltung des Individuums zu erfüllen hat, von so ausserordentlich grosser Wichtigkeit ist. Da nun diese Aufgabe wesentlich in der Erhaltung eines regelmässigen Blutstromes durch die Organe besteht, so werden zweckmässige Herzreflexe im Allgemeinen solche sein, welche eine den Bedürfnissen entsprechende Blutzufuhr ans dem Herzen ins arterielle System gewäbrleisten. Solche Reflexe würden also bei etwaigen Störungen zu erwarten sein.

Da es sich nun aus unseren Versuchen herausgestellt hat, dass von der Kammer aus auf reflectorischem Wege inotrope, chronotrope und dromotrope Wirkungen positiver und negativer Art auf verschiedenen Herzabtheilungen hervorgerufen werden können und jede dieser Wirkungen theils einzeln, theils in verschiedener Weise mit anderen combinirt vorkommen kann, so ist damit jedenfalls die Möglichkeit zweckmässig regulatorischer Effecte bewiesen. Von der Art, Grösse und Dauer und von dem Ort der Störungen in der Kammerwand wird es abhängig sein, welche der möglichen Reflexe oder Reflexcombinationen wirklich zu Stande kommen. Bei künstlichen Störungen, wie in unseren Versuchen mit electrischer Tetanisation der Kammerwand, dürfen wir nicht dieselben Reflexerscheinungen, wie bei Störungen organischer Natur (Entzündung, Entartung, Klappenfehler u. s. w.) erwarten. Sind doch die Reizungen in unseren Versuchen ganz anderer Art, ihre Angriffspunkte und ihre Einwirkungsweise solehe, wie sie unter natürlichen physiologischen oder pathologischen Umständen nie vorkommen. Doch scheint auch in unseren Versuchen den Reflexen im Allgemeinen ein regulatorischer oder compensatorischer Charakter zuzukommen. Der Thätigkeitserhöhung der Kammer, welche in Folge der Einwirkung tetanisirender Ströme auf die Kammermuskeln auftritt und die sich in vielen Fällen sichtbar in Extrasystolen äussert, muss in ihrem Einfluss auf den Blatstrom durch die negativ inotropen Reflexe auf die Vorkammer, welche fast nie vermisst wurden, entgegengewirkt werden. Auch können die sich damit combinirenden negativ chrono- und dromotropen Effecte sich in gleichem Sinn compensatorisch geltend machen. Kurz, man hat Recht zu der Vêrmuthung, dass neben der unlängst in diesem Archiv von Prof. Eng elm an (36) nachgewiesenen myo- 
genen Selbstregulation des Herzens noch eine neurogene Selbstregul irung existirt.

Weitere Betrachtungen über die Bedeutung unserer Reflexe fuir den Organismus könnten verfrïht erscheinen, so lange nicht auch die reflectorischen Wirkungen der Herzreizung auf andere Organe, besonders auf die Blutgefässe, näher untersucht sein werden. Hier wird man an Wooldridge's Versuche und an die über die Funktion des N. depressor vorliegenden Untersuehungen anzuknüpfen haben.

\section{Literatur.}

1) Tumaenzew und J. Dogiel, Zur Lehre über das Nervensystem des Herzens. Archiv für mikroskopische Anatomie. 1890, Bd. 36, p. 483.

2) J. F. H e y mans et L. D e m oor, Etude de l'innervation du coeur des vertébrés à l'aide de la méthode de Golgi. Archives de biologie T. XIII, 1893 , p. 619.

3) J. P. Elias, Hartziekten, afhankelijk van aandoeningen der nervi vagi. Proefschrift, Utrecht 1894.

4) K. $\mathrm{B} \mathrm{er} r \mathrm{r}$ o e ts, Over spontaan gangreen en over de van zenuwlijden afhankelijke veranderingen in de wanden der bloedvaten. Proefschrift, Utrecht 1894.

5) A $1 . S \mathrm{mirnow}$, Ueber die sensiblen Nervenendigungen im Herzen bei Amphibien und Säugethieren. Anatomischer Anzeiger, X. Bd., Nr. 23, 10 Juli 1895 .

6) W. H is und R o m berg, Beiträge zur Herzinnervation. Fortschritte der Medizin. Bd. VIII, 1890, Nr. X, p. 374, und Arbeiten aus der medizinischen Klinik zu Leipzig 1893.

7) W. H is Jr., Die Entwickelung des Herznervensystems bei Wirbelthieren. Abhandlungen der math.-phys. Classe der Kgl. Sächs. Gesellschaft der Wissenschaften. Bd. XVIII, Nr. I. Leipzig 1891.

8) G. K ärschner, Art. Herzthätigkeit. R. Wagner's Handwörterbuch der Physiologie II, 1844, p. 78 .und auch p. 73 und flg.

9) J. B u dge, Artikel: Sympathischer Nerv, mit besonderer Rücksicht auf die Herzbewegung. R. Wagner's Handwörterbuch d. Physiol. III, 1846, p. 425 .

10) M. S chiff, Modus der Herzbewegung. Archiv für physiologische Heilkunde 1X, 1850, p. 27 u. flg.

11) Th. W. Engelmann, Ueber das elektrische Verhalten des thätigen Herzens. Pflüger's Archiv u, s. w. Bd. XVII. Siehe auch: Zittingsverslag der Kon. Acad, van Wetensch. Afd. Natuurk., 24 Nov. 1877. 
Ueber Reflexe von der Herzkammer auf das Herz des Frosches. 353

12) Richard Marchand, Beiträge zur Kenntniss der Reizwelle und Contractionswelle des Herzmuskels. Pflüger's Archiv u. s. w. Bd. XV, 1876, pag. 511.

13) Th. W. Eng e lmann, Das rhythmische Polyrheotom. Pflüger's Arch. u. s. w. Bd. VII, 1892, S. 603-620. Siehe auch: Onderzoekingen, gedaan in het physiologisch Laboratorium te Utrecht (4) II, 1893, pag. 164-191, fig. 8 .

14) -, Beobachtungen und Versuche am suspendirten Herzen. Zweite Abhandlung. Ueber die Leitung der Bewegungsreize im Herzen. Pflüger's Archiv u. s. w. Bd. 56, 1894, p. 149-2029, und: Onderzoekingen physiol. Laborat. Utrecht (1) III, 1895, pag. 101-172, Plaat I, fig. 1 en 2.

15) -, Beobachtungen und Versuche am suspendirten Herzen. Dritte Abhandlung. Refractäre Phase und compensatorische Ruhe a. s. w. Pflüger's Archiv u. s. w. Bd. 59, 1894, p. 309-349, Fig. 17 und 18. - 0nderzoek. physiol. lab. Utrecht (4) III, 1895, p. 407 u. flg., Fig. 17 und 18.

16) A. W. Folkmann, Artikel Nervenphysiologie, R. Wagner's Handwörterbuch der Physiologie II, 1844, p. 617.

17) Th. W. Enge $\mathrm{lm}$ a n n, Ueber reciproke und irreciproke Reizleitung, mit besonderer Beziehung auf das Herz. Pflüger's Archiv u. s. w. Bd. 61, 1895, p. 275-284. - Onderzoek. physiol. lab. Utrecht (4) 1896, p. $41-53$.

18) -, Versuche über irreciproke Reizleitung in Muskelfasern. Pflüger's Archiv u. s. w. Bd. 62, 1896, p. 414, Taf. 4, - Onderzoek. Physiol. lab. Utrecht (4) IV, 1896, p. 54-73, P1. I.

19) L. Pagliani, Ueber die Funktion der Herzganglien, Moleschott's Untersuchungen zur Naturlehre etc. Bd. XI, 1874, p. 364 u. 377.

20) K. $\mathrm{Ka}$ is er, Untersuchungen über die Ursache der Rhythmicität der Herzbewegungen. Zeitschrift für Biologie Bd. XXIX, 1893, p. 203-226, Taf. 4.

21) -, Untersuchungen über die Ursache der Rhythmicität etc. II. Ibidem, Bd. XXX, 1894, p. 279-316, Taf. 3.

22) -, Untersuchungen über die Rhythmicität etc. III. Ibidem, Bd. XXXII, 1895, p. 1-20,-Taf. 1, 2.

23) -, Untersuchungen über die Rhythmicität etc. IV. Ibidem, XXXII, 1895, p. 446-463, Taf. 5, 6.

24) F. Goltz, Vagus und Herz. Virchow's Archiv für pathologische Anatomie etc. XXVI, 1863, p. 1.

25) F. Goltz, Ueber Reflexionen vom und zum Herzen. Königsberger medicinische Jahrbücher III, 1863, p. 271.

26) K. Gurboki, Der Vagus ist auch Empfindungsnerv des Herzens. Pflüger's Archiv Bd. 5, 1872, S. 289-290.

27) Cy on und L udwig, Die Reflexe eines der sensiblen Nerven des Herzens etc. Berichte über die Verhandlungen der Sächs. Gesellsch. der Wissensch. zu Leipzig. Mathematisch-physikalische Classe, 1866, p. 307. 
354 L. J. J. M u skens: Ueb. Reflexe von der Herzkammer auf d. Herz etc.

28) H. $\mathrm{Z}$ w a a $\mathrm{rdem}$ a ke r, Over ischaemie van den hartswand. Proefschrift. Amsterdam, 1883.

29) L. W o oldridge, Ueber die Funktionen der Kammernerven des Sängethierherzens. Archiv f. Anatomie und Physiologie, 1883, p. 522.

30) Citirt bei: Milne Edwards, Leçons sur la physiologie et l'anatomie comparée etc. T. IV, Paris, 1859 , p. 15 u. 135.

31) H. v o n $\mathrm{Zi}$ ems s en, Studien über die normalen Bewegungsvorgänge am menschlichen Herzen, sowie über die mechanische und elektrische Erregbarkeit des Herzens und des N. phrenicus, angestellt an dem freiliegenden Herzen der Catharina Seraphin. Deutsches Archiv f. klinische Medizin Bd. XXX, 1881. 36 Seiten. 2 Taf.

32) H. H u ch a rd, Maladies du Coeur, Paris, 1889.

33) An dré Petit, Traité de Mëdecine, Tome V, Paris, 1893.

34) L. J. J. M uskens, Over reflexen van de hartekamer op het hart van Rana. Dissert. Utrecht 1896. - Onderzoekingen, gedaan in het physiol. laborat. der Utrecht'sche Hoogeschool (4) IV, 1897, S. 111-169, Pl. IV. S. a. Verslag. d. k. Akad, te Amsterdam. 26. September 1896. p. $140-144$.

35) Th. W. Engelmann, Ueber den Einfluss der Systole auf die motorische Leitung in der Herzkammer. Dies Archiv Bd. 62, 1896, p. 543.

36) -, Ueber den Ursprung der Herzbewegungen und die physiologischen Eigenschaften der grossen Herzvenen des Frosches. Dies Archiv. Bd. 65, 1896, p. 109-214. S. a. Ueber myogene Selbstregulirung der Herzthätigkeit. Versl, d. kon. Akad. te Amsterdam. 31. Oktober 1896. 